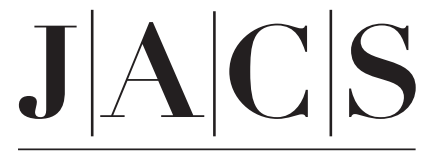

A R T I C L E S

Published on Web 12/30/2003

\title{
Reversible Voltage-Induced Assembly of Au Nanoparticles at Liquid|Liquid Interfaces
}

\author{
Bin Su, ${ }^{\dagger}$ Jean-Pierre Abid, ${ }^{\dagger}$ David J. Fermín, ${ }^{*,+, \neq}$ Hubert H. Girault, ${ }^{\dagger}$ \\ Hana Hoffmannová,§ Petr Krtil,§ and Zdeněk Samec§ \\ Contribution from the Laboratoire d'Electrochimie Physique et Analytique, \\ Institut de Chimie Moléculaire et Biologique, Ecole Polytechnique Fédérale de Lausanne, \\ CH-1015 Lausanne, Switzerland, and J. Heyrovsky Institute of Physical Chemistry, \\ Academy of Science of the Czech Republic, Dolejškova 3, Prague, Czech Republic
}

Received September 19, 2003; E-mail: david.fermin@iac.unibe.ch

\begin{abstract}
The voltage-induced assembly of mercaptosuccinic acid-stabilized Au nanoparticles of $1.5 \pm$ $0.4 \mathrm{~nm}$ diameter is investigated at the polarizable water|1,2-dichloroethane interface. Admittance measurements and quasi-elastic laser scattering (QELS) studies reveal that the surface concentration of the nanoparticle at the liquid|liquid boundary is reversibly controlled by the applied bias potential. The electrochemical and optical measurements provide no evidence of irreversible aggregation or deposition of the particles at the interface. Analysis of the electrocapillary curves constructed from the dependence of the frequency of the capillary waves on the applied potential and bulk particle concentration indicates that the maximum particle surface density is $3.8 \times 10^{13} \mathrm{~cm}^{-2}$, which corresponds to $67 \%$ of a square closedpack arrangement. This system provides a unique example of reversible assembly of nanostructures at interfaces, in which the density can be effectively tuned by the applied potential bias.
\end{abstract}

\section{Introduction}

Current trends in nanosciences and nanotechnology are pointing toward the fabrication of $2 \mathrm{D}$ and $3 \mathrm{D}$ assemblies of monodispersed nanostructures at surfaces. ${ }^{1-3}$ A variety of protocols has been developed in the past few years for the assembly of functionalized metal and semiconductor nanoparticles at electrode surfaces. ${ }^{4-14}$ The optical and electronic

† Ecole Polytechnique Fédérale de Lausanne.

$\doteqdot$ Present address: Departement für Chemie und Biochemie, Universität Bern, Freiestrasse 3, CH-3000 Bern 9, Switzerland.

$\S$ Academy of Science of the Czech Republic.

(1) Gudiksen, M. S.; Lauhon, L. J.; Wang, J.; Smith, D. C.; Lieber, C. M. Nature 2002, 415, 617-620.

(2) Kovtyukhova, N. I.; Mallouk, T. E. Chem.-Eur. J. 2002, 8, 4355-4363.

(3) Maruyama, N. I.; Koito, T.; Nishida, J.; Sawadaishi, T.; Cieren, X.; Ijiro, K.; Karthaus, O.; Shimomura, M. Thin Solid Films 1998, 327-329, 854

(4) Grabar, K. C.; Freeman, R. G.; Hommer, M. B.; Natan, M. J. Anal. Chem. 1995, 67, 735-743.

(5) Grabar, K. C.; Allison, K. J.; Baker, B. E.; Bright, R. M.; Brown, K. R.; Freeman, R. G.; Fox, A. P.; Keating, C. D.; Musick, M. D.; Natan, M. J. Langmuir 1996, 12, 2353-2361.

(6) Grabar, K. C.; Smith, P. C.; Musick, M. D.; Davis, J. A.; Walter, D. G. Jackson, M. A.; Guthrie, A. P.; Natan, M. J. J. Am. Chem. Soc. 1996, 118 , $1148-1153$

(7) Baum, T.; Bethell, D.; Brust, M.; Schiffrin, D. J. Langmuir 1999, 15, 866871.

(8) Chen, S. J. Phys. Chem. B 2000, 104, 663-667.

(9) Hicks, J. F.; Zamorini, F. P.; Murray, R. W. J. Phys. Chem. B 2002, 106, $7751-7757$

(10) Kotov, N. A.; Dekany, I.; Fendler, J. H. J. Phys. Chem. 1995, 99, 1306513069 .

(11) Bakkers, E.; Marsman, A. W.; Jenneskens, L. W.; Vanmaekelbergh, D. Angew. Chem., Int. Ed. 2000, 39, 2297-2299.

(12) Bakkers, E.; Roest, A. L.; Marsman, A. W.; Jenneskens, L. W.; de Jongvan Steensel, L. I.; Kelly, J. J.; Vanmaekelbergh, D. J. Phys. Chem. B 2000, 104, 7266-7272.

(13) Bakkers, E. P. A. M.; Vanmaekelbergh, D. Phys. Rev. B 2000, 62, R7743R7746.

(14) Liu, Y. J.; Wang, Y. X.; Claus, R. O. Chem. Phys. Lett. 1998, 298, 315319

10.1021/ja0386187 CCC: $\$ 27.50$ ㄷ 2004 American Chemical Society properties of the ensemble are determined not only by the properties of individual nanostructures but also by the particleparticle and particle-substrate interactions. ${ }^{7,13,15} \mathrm{~A}$ rather challenging issue is to address the effect of these interactions on the reactivity of individual particles. To assess this information, assembling protocols should be developed in which the interparticle and particle-substrate orbital couplings can be effectively deconvoluted. Recent publications by Dinsmore and co-workers show that interparticle interactions can be uniquely studied at liquid|liquid interfaces. ${ }^{16,17}$

Interfaces between two immiscible electrolyte solutions (ITIES) provide a defect-free junction for studying adsorption phenomena, charge-transfer reactions, and phase-formation processes. ${ }^{18-20}$ In the presence of hydrophilic and hydrophobic electrolytes, the Galvani potential difference between the two liquid phases can be biased potentiostatically. The Debye length at the interfacial region extends over a distance of ca. $10 \mathrm{~nm}$, depending on the concentration of the electrolytes, although controversies still remain concerning the effect of ion-ion interactions in the potential distribution. ${ }^{21}$ Our recent works

(15) Kakkassery, J. J.; Abid, J.-P.; Carrara, M.; Fermín, D. J. Faraday Discuss. 2004, 125, 157-169.

(16) Nikolaides, M. G.; Bausch, A. R.; Hsu, M. F.; Dinsmore, A. D.; Brenner, M. P.; Weitz, D. A. Nature 2002, 420, 299-301.

(17) Lin, Y.; Skaff, H.; Emrick, T.; Dinsmore, A. D.; Russell, T. P. Science 2003, 299, 226-229.

(18) Fermín, D. J.; Jensen, H.; Girault, H. H. In Encyclopedia of Electrochem istry; Calvo, E. J., Eds.; Interfacial Kinetics and Mass Transport, Vol. 2; Wiley-VCH: New York, 2003; pp 360-390.

(19) Fermín, D. J.; Lahtinen, R. In Liquid Interfaces in Chemical, Biological, and Pharmaceutical Applications; Volkov, A., Eds.; Marcel Dekker: Boca Raton, 2001; pp 179-228.

(20) Kakiuchi, T. In Liquid Interfaces in Chemical, Biological, and Pharmaceutical Applications; Volkov, A., Eds.; Marcel Dekker: Boca Raton, 2001; pp $105-121$.

J. AM. CHEM. SOC. 2004, 126, 915-919 - 915 


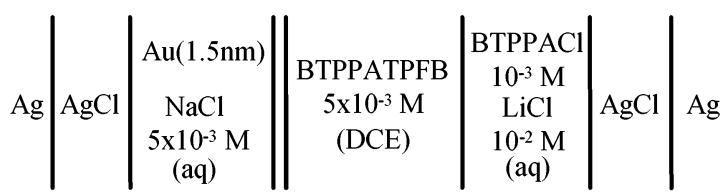

Figure 1. Schematic representation of the electrochemical cell.

based on polarizable water|1,2-dichloroethane interfaces indicated that $\mathrm{TiO}_{2}$ nanoparticles can be assembled at the interface upon applying a potential bias. ${ }^{22,23}$ In the present communication, we shall study the reversible potential-induced assembly of Au particles at ITIES. Differential capacitance and quasielastic laser scattering (QELS) measurements provide clear evidence that the particle density at the liquid|liquid boundary can be tuned by the Galvani potential difference applied between both electrolyte phases. Electrocapillary curves constructed from QELS measurements allow evaluating the particle excess concentration as well as the effective charge of the particles at the interface. No evidence of substantial aggregation of the mercaptosuccinic acid derivatized nanoparticles at the liquid|liquid junction are observed under the experimental conditions employed. The implications of these findings in future strategies for studying interparticle interactions in 2D assemblies are also briefly exposed.

\section{Experimental Section}

Synthesis of the Gold Nanoparticles. Aqueous suspensions of gold nanoparticles protected by a monolayer of mercaptosuccinic acids (MSA) were synthesized by $\mathrm{NaBH}_{4}$ reduction of $\mathrm{AuCl}_{4}{ }^{-}$in a mixture of water and methanol. The synthesis follows the protocol reported by Chen and Kimura, ${ }^{24}$ including 10 consecutive washing and centrifugation steps with $20 \%$ water/methanol (v/v) solution and pure methanol. This sequence ensures the removal of inorganic ions and free MSA. The solvent excess was evaporated at temperatures below $40{ }^{\circ} \mathrm{C}$ and pressure under $5 \times 10^{-3}$ Torr for $12 \mathrm{~h}$. The core size of the resulting particles exhibited an average diameter of $(1.5 \pm 0.4) \mathrm{nm}$ as determined by HRTEM.

Electrochemical Cell. All reagents used were analytical grade. The aqueous and organic solutions were prepared with the ultrapure water from a Milli-Q system (Millipore Milli-Q.185) and 1,2-dichloroethane (DCE) (Fluka 98\% for HPLC), respectively. The compositions of the electrolyte solutions are indicated in Figure 1. The organic supporting electrolyte was bis(triphenylphosphoranylidene) ammonium tetrakis(pentafluorophenyl)borate (BTPPATPFB). ${ }^{25}$ It was prepared by metathesis of bis(triphenylphosphoranylidene)ammonium chloride (BTPPACl) (Fluka, $>98 \%$ for HPLC) and lithium tetrakis(pentafluorophenyl)borate diethyl etherate (LiTPFB) (Boulder Scientific Company, gross wt: $64.3 \mathrm{gm} / \mathrm{tare}$, wt: $54.3 \mathrm{gm} / \mathrm{net}$ ) in a molar ratio of $1: 1$ in $2: 1$ methanol/water mixture, followed by recrystallization in acetone. The geometric interfacial area was $1.53 \mathrm{~cm}^{2}$. The electrochemical experiments were performed using a homemade four-electrode system connected to a Stanford Research System DS335 synthesized function generator. The interfacial capacitance was calculated from admittance measurements using a Stanford Research System SR830 lock-in amplifier at a frequency of $6 \mathrm{~Hz}$ and amplitude of $4 \mathrm{mV} \mathrm{rms}$. The applied potential was referred to the potential of the minimum of the

(21) Samec, Z. In Liquid Interfaces in Chemical, Biological, and Pharmaceutical Applications; Volkov, A., Eds.; Marcel Dekker: Boca Raton, 2001; pp 415437.

(22) Jensen, H.; Fermín, D. J.; Moser, J. E.; Girault, H. H. J. Phys. Chem. B 2002, 106, 10908-10914

(23) Fermín, D. J.; Jensen, H.; Moser, J. E.; Girault, H. H. ChemPhysChem 2003, 4, 85-89.

(24) Chen, S.; Kimura, K. Langmuir 1999, 15, 1075-1082.

(25) Fermín, D. J.; Duong, H. D.; Ding, Z. F.; Brevet, P. F.; Girault, H. H. Phys. Chem. Chem. Phys. 1999, 1, 1461-1467.
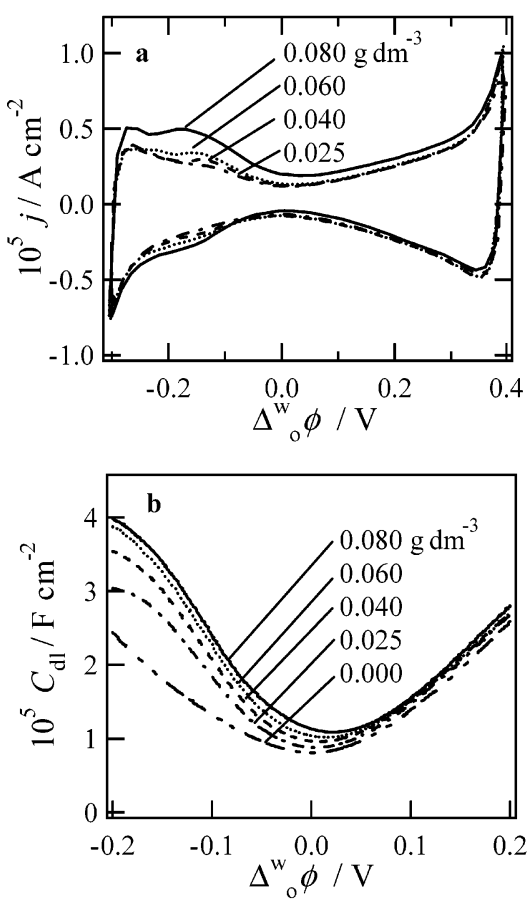

Figure 2. Cyclic voltammograms at $100 \mathrm{mV} \mathrm{s}^{-1}$ (a) and differential capacitance curves (b) for various concentrations of Au nanoparticles. Both electrochemical responses indicate the presence of an excess negative charge at the aqueous side of the liquid|liquid boundary upon negative polarization. The minimum of the capacitance in the absence of the nanoparticles was taken as the pzc.

differential capacitance curves obtained in the absence of the nanoparticle suspension. This potential bias was close to the maximum in the electrocapillary curve, confirming that it corresponds to the potential of zero charge $(p z c)$ at the water|DCE interface.

Interfacial Tension Measurements. Quasi-elastic laser scattering measurements under potentiostatic control were performed in a 15.9 $\mathrm{cm}^{2}$ all-glass cylindrical cell using the optical heterodyne mixing technique. ${ }^{26}$ An optical glass window was placed in contact with the aqueous phase to minimize the diffraction from the water|air interface. A $4 \mathrm{~mW}$ red $\mathrm{He}-\mathrm{Ne}$ laser $(632.8 \mathrm{~nm})$ was perpendicularly directed to the liquid|liquid interface as well as a diffraction grating (local oscillator $\left.^{26}\right)$. Further details of the experimental setup have been described elsewhere. ${ }^{27}$ The grating constant $(d)$ of the set up was determined as $0.358 \mathrm{~mm}$ by the following equation:

$$
d \sin \alpha=n \lambda
$$

where $n$ is the order of the diffraction spot, $\lambda$ is the wavelength of the incident light, and $\alpha$ is the angle of the diffracted beam with respect to the fundamental beam at the detector plane. The third-order diffraction spot was monitored by a photomultiplier tube and was analyzed by a fast-Fourier transform (FFT) analyzer (Stanford Research System SR770). The aqueous and organic solutions were saturated with each other. All of the experiments were carried out at room temperature $(295 \pm 2 \mathrm{~K})$.

\section{Results and Discussion}

Cyclic voltammograms at $100 \mathrm{mV} \mathrm{s}^{-1}$ and various concentrations of the $\mathrm{Au}$ nanoparticles are illustrated in Figure 2a. The voltammograms feature a peak at negative potentials for which position and broadening are slightly dependent on the concentration of the nanoparticles. This response is not observed in

(26) Hard, S.; Hamnerius, Y.; Nilsson, O. J. Appl. Phys. 1976, 47, 2433-2442.

(27) Nagatani, H.; Samec, Z.; Brevet, P. F.; Fermín, D. J.; Girault, H. H. J. Phys. Chem. B 2003, 107, 786-790. 
aqueous solutions containing free $\mathrm{MSA}$ or $\mathrm{AuCl}_{4}{ }^{-}$, indicating that the peak is associated with the MSA-derivatized $\mathrm{Au}$ nanoparticles. The peak current exhibits a linear dependence on the potential scan rate, suggesting that the process is associated with the specific adsorption of the nanoparticles. In addition to the uncompensated resistance effects, the width of adsorption peaks in cyclic voltammograms at the polarizable liquid|liquid interfaces is determined by the charge of the adsorbing species and the effective potential drop in the aqueous side of the junction. ${ }^{28}$ The relatively broad adsorption features in Figure 2a provide indications that (i) the effective charge per particle is significantly smaller than the charge expected if all the mercaptosuccinic acid groups were fully ionized and (ii) only a small portion of the applied potential is developed in the aqueous side of the interface. Additional aspects concerning the effective charge per particle will be discussed further below. Evidence for the adsorption nature of the voltammetric responses can be obtained from the differential capacitance $\left(C_{\mathrm{dl}}\right)$ curves shown in Figure 2b. These curves were estimated from admittance measurements assuming that the impedance of the cell can be expressed in terms of the double-layer capacitance and the uncompensated resistance between the reference electrodes. The value of $C_{\mathrm{dl}}$ can be rationalized as the derivative of the excess charge in the electric double layer with respect to the applied potential.

It can be observed that the excess charge at the interfacial boundary increases at negative Galvani potential difference. In this potential region, the inner potential of the aqueous phase is biased negatively with respect to the organic phase, inducing the accumulation of anions in the diffuse layer of the aqueous side of the interface. We have observed a similar behavior in the presence of $\mathrm{TiO}_{2}$ particles at basic $\mathrm{pH}$ where the oxide surface is negatively charged. ${ }^{22}$ The excess charge associated with the assembly of Au nanoparticle increases with increasing particle concentration up to values close to $0.1 \mathrm{~g} \mathrm{dm}^{-3}$. This behavior further indicates that the excess charge at negative potentials is originating from the polarization-induced assembly of the nanoparticles at the liquid|liquid boundary.

Changes in the interfacial tension due to the formation of the nanoparticle assembly at the water|DCE boundary were estimated from the variations in the QELS power spectrum. The evolution of the power spectrum corresponding to the thirdorder diffracted spot with increasing concentration of the nanoparticles at $\Delta_{\mathrm{o}}{ }^{\mathrm{w}} \phi=-0.16 \mathrm{~V}$ is illustrated in Figure 3. The displacement of the maximum toward lower frequencies indicates a decrease in the frequency of the capillary waves. The relationship between the maximum frequency in the power spectrum $\left(f_{0}\right)$ and the interfacial tension $(\gamma)$ is given by Lamb's equation, ${ }^{29}$

$$
f_{0}=\frac{1}{2 \pi} \sqrt{\frac{\gamma k^{3}}{\rho^{\mathrm{w}}+\rho^{\mathrm{DCE}}}}
$$

where $\rho^{\mathrm{w}}$ and $\rho^{\mathrm{DCE}}$ are the density of water and DCE, respectively. The capillary wavenumber $k$ was estimated assuming that the interfacial tension of the neat water|DCE junction is $28.5 \mathrm{mN} \mathrm{m}^{-1}$. $^{27}$

(28) Nagatani, H.; Fermín, D. J.; Girault H. H. J. Phys. Chem. B 2001, 105, 9463-9473.

(29) Lamb, H. Hydrodynamics, 6th ed.; Cambridge University Press: London, 1932, p 457.

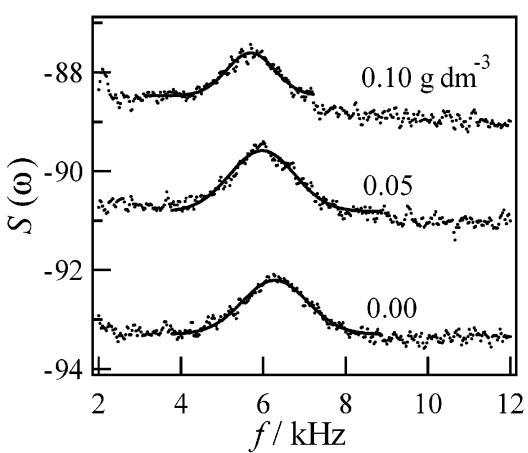

Figure 3. Evolution of the power spectrum corresponding to the thirdorder spot of QELS with increasing concentrations of the Au nanoparticles at $\Delta_{\mathrm{o}}{ }^{\mathrm{w}} \phi=-0.160 \mathrm{~V}$. The decrease of $f_{0}$ with increasing particle concentrations indicates a decrease in the frequency of the capillary waves.

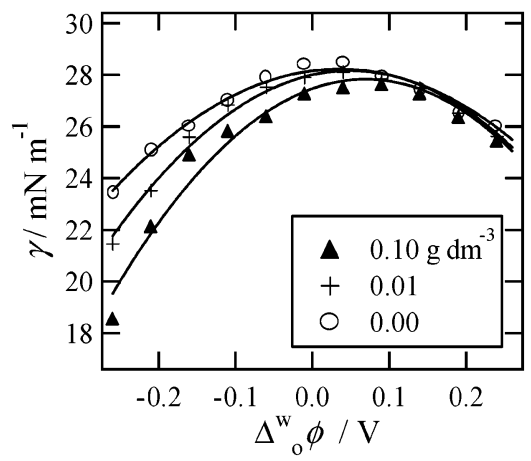

Figure 4. Electrocapillary curves at various particle concentrations, demonstrating that the density of particles at the liquid|liquid interface can be reversibly controlled by the applied bias potential.

The dependence of $f_{0}$ on the concentration of the nanoparticles as well as the potential bias across the interface allowed constructing electrocapillary curves as shown in Figure 4. In the absence of the particles, the maximum of the electrocapillary curves is located at potentials close to the minimum of the differential capacitance. This result confirms that the reference potential employed in this report corresponds to the potential of zero charge. As the concentration of the particles increases, the interfacial tension decreases at negative Galvani potential differences due to the assembly of the Au nanoparticles. Comparison between the results displayed in Figures $2 \mathrm{~b}$ and 4 demonstrates that the onset potential for the formation of the nanoparticle array is close to the $p z c$ at the water|DCE boundary. Furthermore, the dependence of $\gamma$ and $C_{\mathrm{dl}}$ on the applied bias remains unaffected after several potential cycles over the whole polarizable window. These results indicate that the formation of the nanoparticle assembly can be reversibly controlled by the applied voltage and no irreversible processes such as aggregation or gold nucleation appear to take place at the liquid|liquid interface.

To rationalize the changes in the surface tension, it is considered that the charged species distributed in the electrical double layer comprise the ions of the aqueous supporting electrolyte $\mathrm{Na}^{+}$and $\mathrm{Cl}^{-}$, the nanoparticles $\mathrm{Au}^{z-}$ with the charge number $z^{-}(z>0)$, and the ions of the organic supporting electrolyte $\mathrm{BTTPA}^{+}$and $\mathrm{TPFB}^{-}$. As the charge of the MSAderivatized particles is counter-balanced by sodium ions, changes in the concentration of the particles do not affect the $\mathrm{pH}$ significantly. Consequently, the Gibbs adsorption equation at constant temperature $(T)$ and pressure $(P)$ can be expressed as, 


$$
\begin{array}{r}
-d \gamma_{T, P}=\Gamma_{\mathrm{Au}^{-}} d \tilde{\mu}_{\mathrm{Au}^{z^{-}}}+\Gamma_{\mathrm{Na}^{+}} d \tilde{\mu}_{\mathrm{Na}^{+}}+\Gamma_{\mathrm{Cl}^{-}} d \tilde{\mu}_{\mathrm{Cl}^{-}}+ \\
\Gamma_{\mathrm{BTTPA}^{+}} d \tilde{\mu}_{\mathrm{BTPPA}^{+}}+\Gamma_{\mathrm{TPFB}^{-}} d \tilde{\mu}_{\mathrm{TPFB}^{-}}
\end{array}
$$

where $\gamma$ is the interfacial tension and $\tilde{\mu}$ and $\Gamma$ are the electrochemical potentials and the surface excess concentrations, respectively. Upon expressing the changes in the chemical potentials of the electrolytes $\mathrm{NaCl}, \mathrm{Na}_{z} \mathrm{Au}$, and BTPPATPFB as,

$$
\begin{aligned}
d \mu_{\mathrm{Na}_{z} \mathrm{Au}} & =z d \tilde{\mu}_{\mathrm{Na}^{+}}+d \tilde{\mu}_{\mathrm{Au}_{z}{ }^{-}} \\
d \mu_{\mathrm{NaCl}} & =d \tilde{\mu}_{\mathrm{Na}^{+}}+d \tilde{\mu}_{\mathrm{Cl}^{-}} \\
d \mu_{\mathrm{BTPATPFB}} & =d \tilde{\mu}_{\mathrm{BTPPA}^{+}}+d \tilde{\mu}_{\mathrm{TPFB}^{-}}
\end{aligned}
$$

the electrocapillary equations is obtained,

$$
\begin{aligned}
-d \gamma_{T, P}= & Q^{\mathrm{w}} d \Delta_{\mathrm{o}}^{\mathrm{w}} \phi+\Gamma_{\mathrm{Au}_{z}} d \mu_{\mathrm{Na}_{z} \mathrm{Au}}+ \\
& \left(\Gamma_{\mathrm{Na}^{+}}-z \Gamma_{\mathrm{Au}_{z}-}\right) d \mu_{\mathrm{NaCl}}+\Gamma_{\mathrm{TPFB}^{-}} d \mu_{\mathrm{BTPPATPFB}}
\end{aligned}
$$

where $Q^{\mathrm{w}}$ is the excess charge density on the aqueous side of the interface,

$$
\begin{aligned}
Q^{\mathrm{W}} / F= & \Gamma_{\mathrm{Na}^{+}}-\Gamma_{\mathrm{Cl}^{-}}-z \Gamma_{\mathrm{Au}_{z}^{-}}=-Q^{\circ} / F= \\
\Gamma_{\mathrm{TPFB}^{-}}- & \Gamma_{\mathrm{BTPPA}^{+}}
\end{aligned}
$$

From eq 7, it follows that $Q^{\mathrm{w}}$ and $\Gamma_{\mathrm{Au}_{z}}{ }^{-}$are given by,

$$
Q^{\mathrm{w}}=-\left(\frac{\partial \gamma}{\partial \Delta_{\mathrm{o}}^{\mathrm{w}} \phi}\right)_{\mathrm{T}, \mathrm{P}, u_{i}}
$$

and

$$
\Gamma_{\mathrm{Au}_{z}^{-}}=-\left(\frac{\partial \gamma}{\partial \mu_{\mathrm{Na}_{z} \mathrm{Au}}}\right)_{\mathrm{T}, \mathrm{P}, \Delta_{\mathrm{o}} \mathrm{w} \phi, \mu_{\mathrm{NaC},}, \mu_{\mathrm{BTPATPFB}}}
$$

Finally, assuming that the most important contribution to $Q^{\mathrm{w}}$ arises from the $\mathrm{Au}$ nanoparticles, analysis of the electrocapillary curves on the basis of eqs 9 and 10 can provide information on the particle charge number $z$.

The dependencies of $Q^{\mathrm{w}}$ and $\Gamma_{\mathrm{Au}^{2-}}$ on the concentration of the particles in the aqueous suspension as derived from eqs 9 and 10 are illustrated in Figure 5,a and b, respectively. The derivatives were calculated by interpolating the surface tension values employing a polynomial expression. It can be observed that both interfacial parameters increase as the concentration of the particles increases and as the interface is biased to negative potentials. Considering,

$$
Q^{\mathrm{w}} \approx Q^{\mathrm{Au}^{z^{-}}}=-z e \Gamma_{\mathrm{Au}^{--}}
$$

the effective average value of $z$ obtained from the data illustrated in Figure 5 corresponds to $5.1 \pm 0.7$. This value appears rather low, considering that particles of this diameter can accommodate at least 50 thiolate ligands. ${ }^{30}$ The carboxyl groups in MSA exhibit two $\mathrm{p} K_{\mathrm{a}}$ values at 4.90 and $5.64 \cdot{ }^{24}$ consequently, these groups should be fully ionized at the $\mathrm{pH}$ of the aqueous electrolyte $(\mathrm{pH}=7)$. To verify the estimation of the average

(30) Hostetler, M. J.; Wingate, J. E.; Zhong, C. J.; Harris, J. E.; Vachet, R. W.; Clark, M. R.; Londono, J. D.; Green, S. J.; Stokes, J. J.; Wignall, G. D.; Glish, G. L.; Porter, M. D.; Evans, N. D.; Murray, R. W. Langmuir 1998, 14, 17-30.
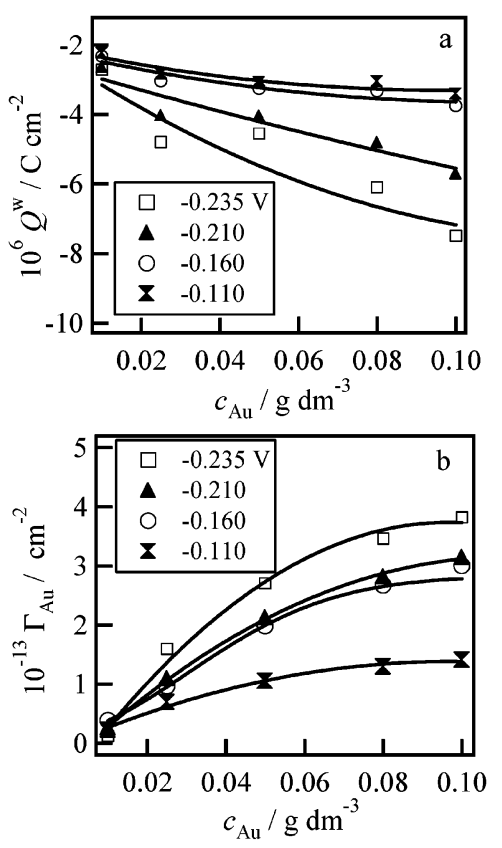

Figure 5. Charge density (a) and surface excess of the particles (b) as a function of the bulk concentration of the nanoparticles for various Galvani potential differences.

charge per particle, capillary electrophoresis was performed on nanoparticle suspensions at $\mathrm{pH} 7 .{ }^{31}$ Our preliminary results yielded a mobility of $(4.4 \pm 0.1) \times 10^{-8} \mathrm{~m}^{2} \mathrm{~V}^{-1} \mathrm{~s}^{-1}$, which corresponds to a value of $z=3.9 \pm 0.1$, assuming spherical nanoparticles with an average diameter of $1.5 \mathrm{~nm}$. Templeton et al. have also obtained similar average charges for Au particles of the same dimensions stabilized by Tioponin. ${ }^{32}$ This $z$ value is consistent with the previous estimations based on the electrocapillary curves, confirming that the changes in the surface tension are related to the interfacial assembly of the $\mathrm{Au}$ particles.

The previous thermodynamic analysis does not establish a model a priori for the structure of the nanoparticle assembly. The maximum value of $\Gamma_{\mathrm{Au}^{2-}}$ estimated from the electrocapillary curves is $3.8 \times 10^{13} \mathrm{~cm}^{-2}$, corresponding to approximately $67 \%$ of a square closed-pack arrangement of spherical particles of $1.5-\mathrm{nm}$ diameter in a pseudo-2D assembly. In principle, the particle assembly can be visualized as a concentration polarization phenomenon, i.e., the particles are distributed in the spacecharge region at the aqueous side of the interface. However, it should be also taken into account that the diameter of the particles is comparable to the characteristic Debye length at the aqueous side of the interface, i.e., $5 \mathrm{~nm}$ for the electrolyte composition described in Figure 1. Consequently, although the experimental results do not provide unambiguous evidences of the formation of a 2D assembly upon polarization, the intrinsic structure of the interface determines that the particles are confined to a narrow region within few nanometers from the liquid|liquid boundary.

In summary, we have provided evidences that assembly of Au nanoparticles can be onset at the liquid|liquid boundary upon applying a potential bias. This approach has the unique

(31) Experiments were performed in a 60-cm long capillary, with an applied voltage of $30 \mathrm{kV}$ and a UV-visible detector set at $200 \mathrm{~nm}$.

(32) Templeton, A. C.; Cliffel, D. E.; Murray, R. W. J. Am. Chem. Soc. 1999, 121,7081-7089.

918 J. AM. CHEM. SOC. - VOL. 126, NO. 3, 2004 
advantage of effectively controlling the number of particles at the interface by tuning the Galvani potential difference. In conjunction with surface spectroscopic techniques, this method allows studying single vs collective properties of metal and semiconductor nanoparticles. We also expect to address questions regarding the potential distribution across the liquid|liquid boundary via examining the interfacial behavior of the nanoparticles of different sizes.

Acknowledgment. The present work is partly financed by Fonds National Suisse de la Recherche Scientifique (Project 2000-067050.01) and the Office Fédéral de l'Education et de la Science (Project C02.0010). H.H. and Z.S. gratefully acknowledge Visiting Fellowships from the EPFL and the financial support form the Grant Agency of the Czech Republic (No. 203/01/0946). We are indebted to Michel Carrara and Xiaoxia Bai for the capillary electrophoresis measurements and to Valérie Devaud for the technical assistance. We also thank Joseph J. Kakkassery and Nicolas Eugster for the fruitful discussions. The Laboratoire d'Electrochimie Physique et Analytique is part of the TMR network SUSANA (Supramolecular Self-Assembly of Interfacial Nanostructures).

JA0386187 